Research Article

\title{
Use of Network Pharmacology to Explore the Mechanism of Gegen (Puerariae lobatae Radix) in the Treatment of Type 2 Diabetes Mellitus Associated with Hyperlipidemia
}

\author{
Guozhen Yuan $\mathbb{D}$, Shuai Shi $\mathbb{D}$, Qiulei Jia $\mathbb{D}$, Jingjing Shi $\mathbb{D}$, Shuqing Shi $\mathbb{D}$,

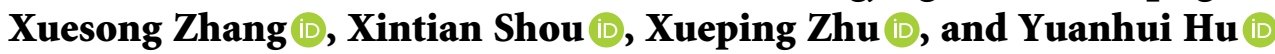 \\ Department of Cardiology, Guang'anmen Hospital, China Academy of Chinese Medical Sciences, Beijing, China \\ Correspondence should be addressed to Yuanhui Hu; huiyuhui55@sohu.com
}

Received 5 November 2020; Revised 5 March 2021; Accepted 6 April 2021; Published 17 April 2021

Academic Editor: Daniel Dias Rufino Arcanjo

Copyright $\odot 2021$ Guozhen Yuan et al. This is an open access article distributed under the Creative Commons Attribution License, which permits unrestricted use, distribution, and reproduction in any medium, provided the original work is properly cited.

Rapid increases in metabolic disorders, such as type 2 diabetes mellitus (T2DM) and hyperlipidemia, are becoming a substantial challenge to worldwide public health. Traditional Chinese medicine has a long history and abundant experience in the treatment of diabetes and hyperlipidemia, and Puerariae lobatae Radix (known as Gegen in Chinese) is one of the most prevalent Chinese herbs applied to treat these diseases. The underlying mechanism by which Gegen simultaneously treats diabetes and hyperlipidemia, however, has not been clearly elucidated to date. Therefore, we systematically explored the potential mechanism of Gegen in the treatment of T2DM complicated with hyperlipidemia based on network pharmacology. We screened the potential targets of Gegen, T2DM, and hyperlipidemia in several online databases. Then, the hub targets were analyzed by performing protein-protein interaction, Gene Ontology (GO), and Kyoto Encyclopedia of Genes and Genomes (KEGG) enrichment assays, and finally, the complicated connections among compounds, targets, and pathways were visualized in Cytoscape. We found that isoflavones, including daidzein, genistein, and puerarin, as well as $\beta$-sitosterol, are the key active ingredients of Gegen responsible for its antidiabetic and antihyperlipidemia effects, which mainly target AKR1B1, EGFR, ESR, TNF, NOS3, MAPK3, PPAR, CYP19A1, INS, IL6, and SORD and multiple pathways, such as the PI3K-Akt signaling pathway; the AGE-RAGE signaling pathway in diabetic complications, fluid shear stress, and atherosclerosis; the PPAR signaling pathway; insulin resistance; the HIF1 signaling pathway; the TNF signaling pathway; and others. These active ingredients also target multiple biological processes, including the regulation of glucose and lipid metabolism, the maintenance of metabolic homeostasis, and anti-inflammatory and antioxidant pathways. In conclusion, Gegen is a promising therapeutic phytomedicine for T2DM with hyperlipidemia that targets multiple proteins, biological processes, and pathways.

\section{Introduction}

The rapidly increasing incidence of chronic metabolic diseases has become a global health threat, among which diabetes mellitus (DM) and hyperlipidemia must be mentioned. First, the latest global estimate from the International Diabetes Federation is that 463 million people suffered from diabetes mellitus in 2019 and that the number will be 700 million by 2045 [1]. At the same time, approximately $90 \%$ of cases of type 2 diabetes mellitus (T2DM) are diagnosed [2]. In addition, hyperlipidemia is a wellknown risk factor for cardiovascular and cerebrovascular diseases such as atherosclerosis, coronary heart disease, and stroke [3-6]. The morbidity of lipid disorders remains high in both developing and developed countries. In Western Europe and the United States, at least one-third of the population has hypercholesterolemia [7, 8]. In China, the overall prevalence of dyslipidemia in adults was $40.4 \%$ in 2012 [9]. Moreover, these two metabolic disorders are both closely related to cardiovascular disease [10]. The majority of patients with diabetes mellitus and/or hyperlipidemia ultimately succumb to heart or blood vessel disease $[11,12]$.

In fact, the two groups often overlap; namely, someone may suffer from both diabetes and hyperlipidemia. 
Compared with individuals without dyslipidemia, the risk for diabetes in subjects with hyperlipidemia increases approximately 2 - to 3 -fold [13]; more than $75 \%$ of patients with T2DM have lipid disorders [14]. Diabetes mellitus and lipoproteins are highly interregulated, and thus the complex relationship between diabetes and hyperlipidemia remains unclear [15]. Undoubtedly, once one metabolic disorder occurs, a higher risk of other complications exists $[13,16]$, and the prevalence of cardiovascular disease is higher in patients with metabolic disorder [17].

Although many drugs have been successfully applied to treat T2DM and hyperlipidemia, safe and effective medicines targeting both diseases at the same time are still lacking. 3-Hydroxy-3-methylglutaryl-coenzyme A reductase inhibitors (also known as statins), the most widely used lipid-lowering drugs in the clinic, have consistently been reported to cause new-onset diabetes mellitus [18]. In addition, the management of complications of these diseases is still a major challenge in clinical practice and a substantial global healthcare burden [19-21].

As an effective supplementary and alternative medicine, traditional Chinese medicine (TCM) has attracted increasing attention. Chinese medicinal herbs are regarded as a rich source for natural drug development. Gegen, the dried root of the leguminous plant Pueraria lobata (Willd.) Ohwi or Pueraria thomsonii Benth., is a very popular Chinese herb that has been used as a medicine and food. From the perspective of TCM theory, Gegen has the pharmacological functions of clearing heat and promoting the secretion of saliva and body fluid. In clinical practice, Gegen is one of the commonly used herbs for the treatment of metabolic and cardiovascular diseases, such as diabetes mellitus and hyperlipidemia [22, 23]. Some studies on the effects of Gegen-containing formulas (such as Gegen Qinlian Decoction) and Gegen extracts (such as puerarin) on metabolic disturbances were performed [22, 24], but no one has reported the mechanism by which Gegen acts on T2DM complicated with hyperlipidemia to date.

In addition, the rapid development of computer technology enables the identification of the targets and mechanisms of multicomponent natural herbs, accelerating the process of drug development and application because of its low cost and high efficiency [25, 26]. Accordingly, we applied network pharmacology to systematically explore the potential mechanism of Gegen for treating T2DM associated with hyperlipidemia in an attempt to find a novel and beneficial therapy for this increasingly prevalent concurrent metabolic disorder.

\section{Materials and Methods}

2.1. Screening the Active Ingredients of Gegen. Active components of Gegen were selected from the Traditional Chinese Medicine Systems Pharmacology (TCMSP) Database (https://tcmspw.com/tcmsp.php) according to oral bioavailability $(\mathrm{OB}) \geq 30 \%$ and drug-likeness $(\mathrm{DL}) \geq 0.18$, two parameters of ADME (absorption, distribution, metabolism, and excretion) properties for evaluating compounds. Furthermore, ingredients not meeting the screening criteria but reported as metabolic regulators were also included by performing text mining.
2.2. Predicting the Targets of the Compounds. The canonical simplified molecular input line entry specification (SMILES) of each compound was retrieved from the PubChem database (https://pubchem.ncbi.nlm.nih.gov/) containing the chemical structures of small organic molecules and information on their biological activities. Then, targets of active ingredients were searched in Binding DB (http://bindingdb. org/bind/index.jsp), DrugBank (https://go.drugbank.com/), STITCH (http://stitch.embl.de/), and Swiss Targets Prediction (http://www.swisstargetprediction.ch/) according to the SMILES formula. The target prediction algorithms of these databases are mainly based on the structural features of small-molecule ligands, namely, the chemical structure similarity of compounds.

2.3. Predicting Targets of Diseases. "Type 2 diabetes mellitus" and "hyperlipidemia" were entered into OMIM (https:// www.omim.org/) and GeneCards (https://www.genecards. org/), respectively, to obtain targets of the diseases. The higher the relevance score of the target predicted in GeneCards, the closer the target to the disease. If too many targets are forecasted, those with scores greater than the median score are empirically considered potential targets. Notably, most proteins and genes have multiple names, such as official names and generic names, and thus their names need to be converted uniformly. The protein targets of compounds were checked in UniProt (https://www.uniprot. org/), an online database that collects protein functional information with accurate, consistent, and rich annotations and was represented as the names of the genes encoding them. Next, all gene names were rechecked in the NCBI gene database (https://www.ncbi.nlm.nih.gov/gene) and converted into Entrez IDs and gene symbols. Repeated targets identified due to nonstandard naming were eliminated. The species of the acquired and checked targets was limited to "Homo sapiens."

2.4. PPI Network Construction and Module Extraction. A protein-protein interaction (PPI) analysis of targets was performed to explore the relationship among the targets and the biological processes involved. A Venn diagram of targets of Gegen, T2DM, and hyperlipidemia was drawn using an online visualization tool (https://hiplot.com.cn/), and the intersections were regarded as hub genes, namely, the potential targets of Gegen working on type 2 diabetes with hyperlipidemia. The hub genes were analyzed using String (http://string-db.org/, version 11.0), an online database that integrates experiments, databases, and text mining data for PPI prediction and extraction, with the organism restricted to "Homo sapiens" and a confidence score $>0.4$. Isolated nodes were hidden, namely, proteins without any interaction. The result was exported as a "TSV" format file and imported into Cytoscape, which is an open-source software project for visualizing any network of molecular components and interactions to construct a PPI network [27]. However, the interpretation of a PPI network is quite difficult because of its complexity, and therefore a suitable auxiliary network analysis tool is needed. MCODE is a plug- 
in of Cytoscape for extracting highly interconnected regions of a network called modules or communities, also known as subnetworks [28]. The module or community is considered a cluster of biological functions, more specifically, protein complexes involved in biological processes as a whole or functional module, such as proteins of the same signaling pathway. Combined with a Gene Ontology (GO) enrichment analysis, the key targets and their biological processes of the network can be predicted, making the explanation of the PPI network more convenient and precise $[29,30]$.

2.5. GO and KEGG Pathway Enrichment Analyses. GO and Kyoto Encyclopedia of Genes and Genomes (KEGG) are both common approaches used to find shared functions among genes based on biological ontologies [31]. Briefly, GO annotates genes to biological processes, molecular functions, and cellular components in a directed acyclic graph structure, and KEGG annotates genes to pathways. ClusterProfiler, a useful tool for gene classification and enrichment analysis, and org.Hs.eg.db, a widely used species annotation package, were run in R4.0, an open-source programming environment, with the strict cutoff of $P$ values $<0.05$ for GO and KEGG enrichment [32-34].

2.6. Compound-Target-Pathway Network Construction. Cytoscape was used to construct and analyze a three-layer network in order to understand the complex relationships among compounds, targets, and pathways. Taking advantage of another built-in network analyzer [35], the topological parameters of active ingredients, targets, and pathways were calculated, including the degree, betweenness centrality (BC), and closeness centrality (CC), which helped to forecast the main components and core targets of Gegen.

\section{Results}

3.1. Active Ingredients of Gegen. Twelve active ingredients of Gegen were finally included based on ADME attributes and text mining. They are shown in Table 1 and include formononetin, daidzein, genistein, and puerarin.

3.2. Targets of Compounds. Targets of active ingredients retrieved from Binding DB, DrugBank, STITCH, and Swiss Targets Prediction (only targets with probability $>0$ included) were merged by deduplication. Ultimately, we obtained 304 targets of the 12 compounds (see Supplementary Material 1 for more details).

3.3. Targets of Diseases. Numerous targets of T2DM and hyperlipidemia were retrieved from the GeneCards database. As mentioned above, we empirically excluded some redundant targets based on their relevance score. Then, by merging the targets from the two disease databases, we obtained 2620 targets for T2DM and 706 for hyperlipidemia (see Supplementary Material 2 for more details).
TABLE 1: Active ingredients and ADME parameters of Gegen.

\begin{tabular}{lcccc}
\hline No. & MOL ID & Molecule name & OB (\%) & DL \\
\hline M1* & MOL001999 & Scoparone & 74.75 & 0.09 \\
M2 & MOL000392 & Formononetin & 69.67 & 0.21 \\
M3 & MOL002959 & 3'-Methoxydaidzein & 48.57 & 0.24 \\
M4 & MOL003629 & Daidzein-4,7-diglucoside & 47.27 & 0.67 \\
M5 & MOL000358 & Beta-sitosterol & 36.91 & 0.75 \\
M6* & MOL012297 & Puerarin & 24.03 & 0.69 \\
M7* & MOL000390 & Daidzein & 19.44 & 0.19 \\
M8* & MOL000481 & Genistein & 17.93 & 0.21 \\
M9* & MOL000663 & Lignoceric acid & 14.9 & 0.33 \\
M10* & MOL009720 & Daidzin & 14.32 & 0.73 \\
M11* & MOL000441 & Lupenone & 11.66 & 0.78 \\
M12* & MOL000391 & Ononin & 11.52 & 0.78 \\
\hline
\end{tabular}

Notes: * The compounds do not meet the inclusion criteria based on ADME $(\mathrm{OB} \geq 30 \%$ and $\mathrm{DL} \geq 0.18)$ but have been reported to have metabolic regulatory effects. Abbreviations: $\mathrm{OB}$, oral bioavailability; DL, druglikeness.

3.4. PPI Network and PPI Modules. A Venn diagram (Figure 1(a)) was drawn for the targets of Gegen, T2DM, and hyperlipidemia, and 65 common targets were obtained (Table 2). These targets were submitted to STRING 11.0 for the PPI analysis, and the result was visualized using Cytoscape, as shown in Figure 1(b). The PPI network has far more edges than expected, indicating that the proteins are at least partially biologically connected as a group. The entire network is highly interactive. Ins (degree $=57$ ) is the core target in the network, since it interacts with almost all other targets. In addition, high-degree targets are mainly distributed in cholesterol metabolism (PPAR- $\gamma$, APOB, and LDLR), inflammation (IL6, TNF, VEGFA, NOS3, CCL2, IL1B, and VCAM1), and oxidative stress (MAPK3, NOS3, and CAT).

Modules were extracted from the PPI network using MCODE, and the 2 modules with the highest scores are displayed in Figures 1(c) and 1(d). Combined with the GO enrichment analysis, the primary biological processes in the PPI network and modules were selected according to the false discovery rate to describe their biological functions. The outcomes show that the primary biological process of the PPI network is the same as that of module 1, namely, the response to oxygen-containing compounds (GO: 1901700). The primary biological process of module 2 is the regulation of cholesterol storage (GO: 0010885).

3.5. GO Enrichment Analysis of the Targets. Biological process (BP, GO: 0008150), cellular component (CC, GO: 0005575), and molecular function (MF, GO: 0003674) enrichment analyses of 65 common targets were performed using the ClusterProfiler package in R. The top 20 terms significantly enriched in $\mathrm{BP}, \mathrm{CC}$, and $\mathrm{MF}$ are shown in Figure $2(P<0.05, P$ values were corrected by the BenjaminiHochberg procedure). The largest number of BP terms was enriched at 1286. Almost all of the top 20 BPs are involved in the regulation of metabolic processes. In addition, regulation of the inflammatory response is also noteworthy. MF terms are second in number (79) and are mainly related to the activity of various receptors and enzymes, as well as molecular binding. CC terms are minimal (38), and the action 


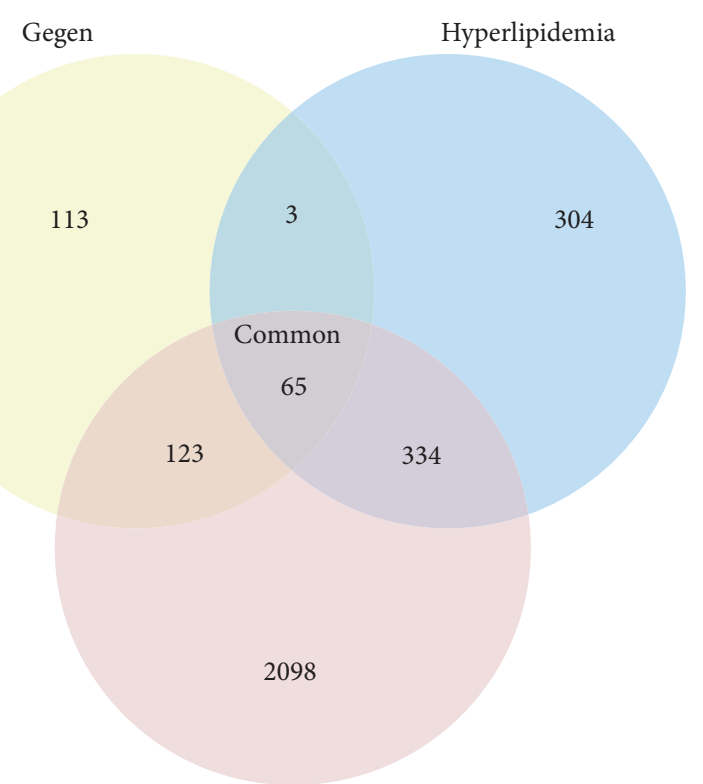

Type 2 diabetes mellitus

(a)

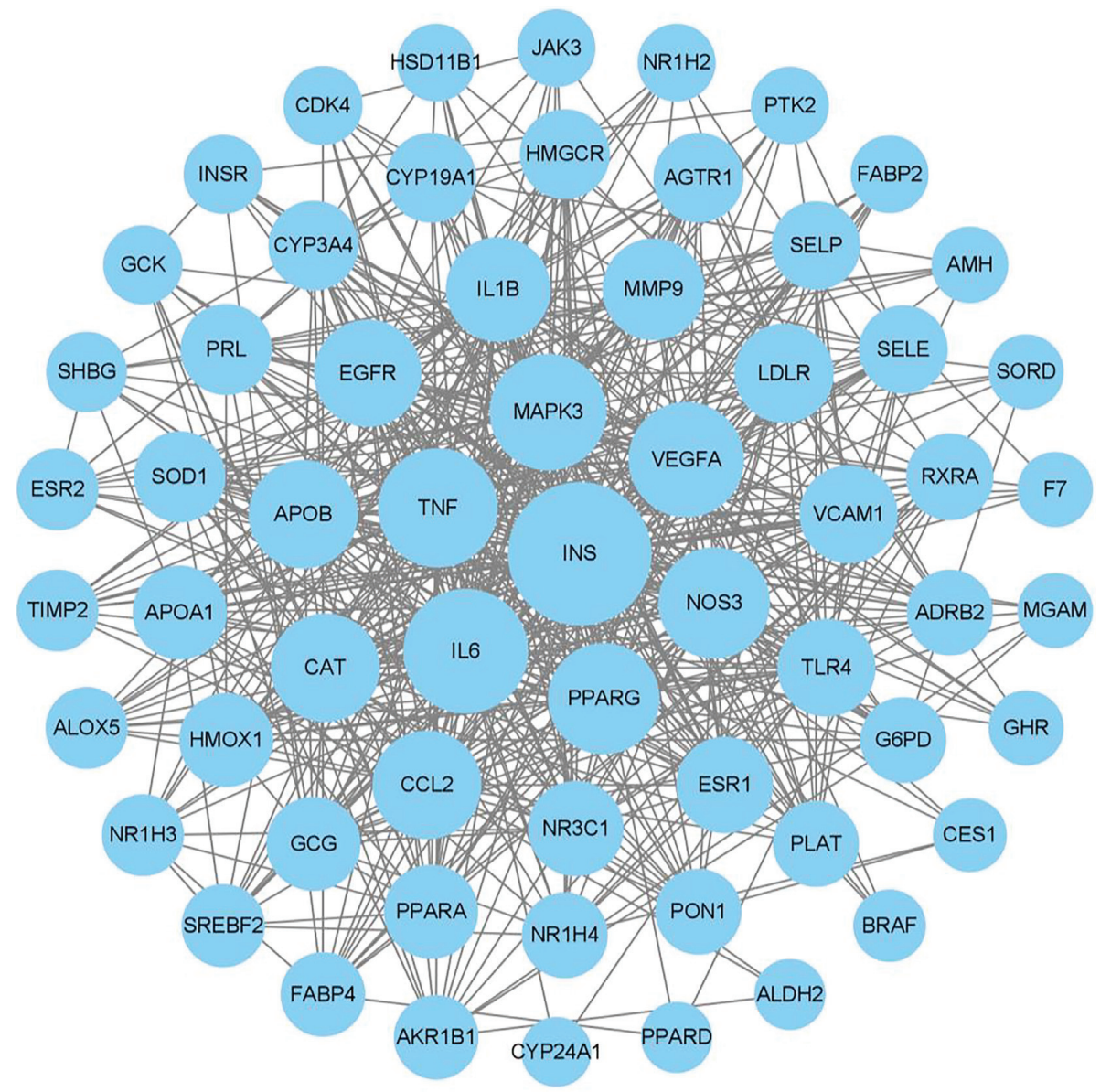

(b)

FIgURE 1: Continued. 


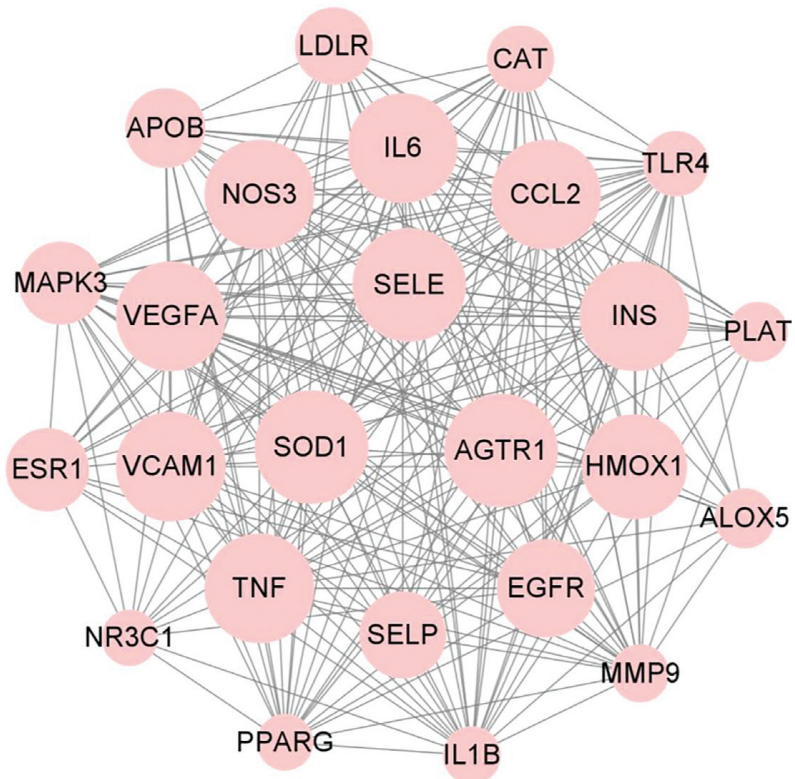

(c)

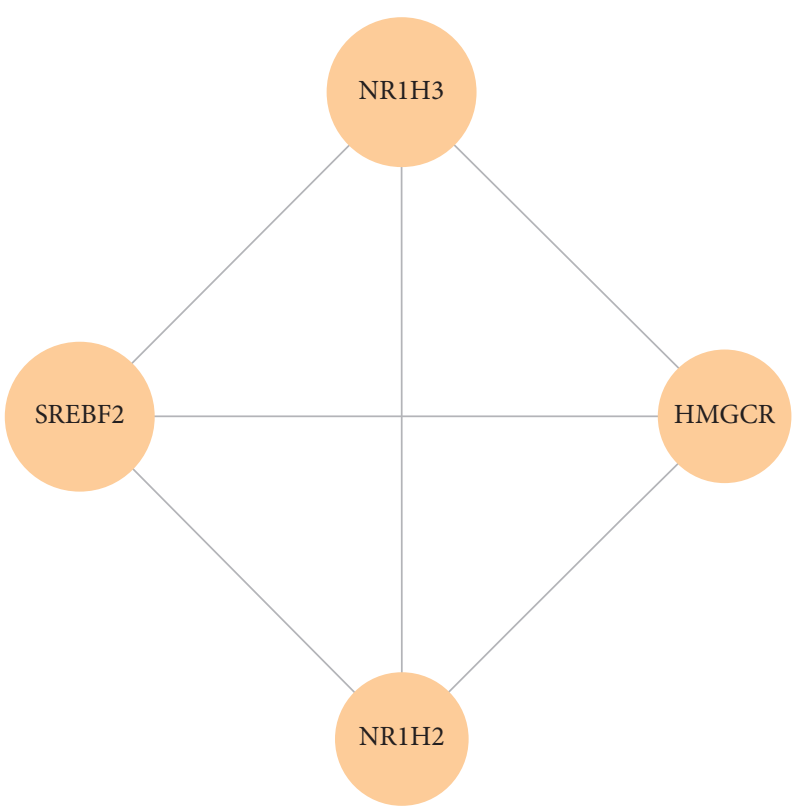

(d)

Figure 1: (a) Venn diagram representing the gene targets among Gegen, T2DM, and hyperlipidemia. (b) PPI network of common targets among Gegen, T2DM, and hyperlipidemia, containing 63 nodes and 538 edges. Each node represents a protein produced by a single proteincoding gene locus. An edge represents the interaction between proteins. The greater the number of edges connected to the same node (namely, the greater the degree), the larger the size of the node. (c) Module of the PPI network with the highest score (module 1), containing 25 nodes and 232 edges. (d) Module of the PPI network with the second highest score (module 2), containing 4 nodes and 6 edges. The higher the MCODE score of the node, the larger the size of the node. The MCODE score reflects the density of the node and surrounding nodes. Abbreviations: T2DM, type 2 diabetes mellitus; PPI, protein-protein interaction.

sites of gene products are mainly located in various types of vesicles, lumens, membranes, and lipoprotein particles or complexes (see Supplementary Material 3 for more details).

3.6. Compound-Target-Pathway Network. The complicated interactions among active components of Gegen, targets, and pathways were visualized with Cytoscape, as shown in
Figure 3. By analyzing this three-layer network based on network topology, the degree, BC, and CC of daidzein are 30 , 0.0498 , and 0.4840 , respectively; thus, daidzein is predicted to be the main bioactive component of Gegen in the treatment of T2DM complicated with hyperlipidemia, followed by genistein (degree $=28, \quad \mathrm{BC}=0.0454$, and $\mathrm{CC}=0.4740$ ), puerarin (degree $=21, \mathrm{BC}=0.0285$, and $\mathrm{CC}=0.4417)$, and $\beta$-sitosterol $($ degree $=19, \mathrm{BC}=0.0207$, and 
TABLE 2: Common targets of Gegen, type 2 diabetes mellitus, and hyperlipidemia.

\begin{tabular}{|c|c|c|c|}
\hline Entrez ID & Gene symbol & Uniprot ID & Protein name \\
\hline 154 & ADRB2 & P07550 & Beta-2 adrenergic receptor \\
\hline 185 & AGTR1 & P30556 & Type-1 angiotensin II receptor \\
\hline 231 & AKR1B1 & $\mathrm{P} 15121$ & Aldo-keto reductase family 1 member $\mathrm{B} 1$ \\
\hline 217 & ALDH2 & P05091 & Aldehyde dehydrogenase, mitochondrial \\
\hline 240 & ALOX5 & P09917 & Polyunsaturated fatty acid 5-lipoxygenase \\
\hline 268 & $\mathrm{AMH}$ & P03971 & Muellerian-inhibiting factor \\
\hline 335 & APOA1 & P02647 & Apolipoprotein A-I \\
\hline 338 & APOB & P04114 & Apolipoprotein B-100 \\
\hline 673 & BRAF & P15056 & Serine/threonine-protein kinase B-raf \\
\hline 847 & CAT & P04040 & Catalase \\
\hline 6347 & CCL2 & P13500 & C-C motif chemokine 2 \\
\hline 1019 & CDK4 & P11802 & Cyclin-dependent kinase 4 \\
\hline 1066 & CES1 & $\mathrm{P} 23141$ & Liver carboxylesterase 1 \\
\hline 1588 & CYP19A1 & P11511 & Aromatase \\
\hline 1591 & CYP24A1 & Q07973 & 1,25-Dihydroxyvitamin $\mathrm{D}(3)$ 24-hydroxylase, mitochondrial \\
\hline 1564 & CYP2D7 & A0A087X1C5 & Putative cytochrome P450 2D7 \\
\hline 1576 & CYP3A4 & P08684 & Cytochrome P450 3A4 \\
\hline 1798 & DPAGT1 & Q9H3H5 & UDP-N-acetylglucosamine-dolichyl-phosphate $\mathrm{N}$-acetylglucosaminephosphotransferase \\
\hline 1956 & EGFR & P00533 & Epidermal growth factor receptor \\
\hline 2099 & ESR1 & $\mathrm{P} 03372$ & Estrogen receptor \\
\hline 2100 & ESR2 & Q92731 & Estrogen receptor beta \\
\hline 2155 & F7 & P08709 & Coagulation factor VII \\
\hline 2169 & FABP2 & P12104 & Fatty acid-binding protein, intestinal \\
\hline 2167 & FABP4 & P15090 & Fatty acid-binding protein, adipocyte \\
\hline 2539 & G6PD & $\mathrm{P} 11413$ & Glucose-6-phosphate 1-dehydrogenase \\
\hline 2641 & GCG & $\mathrm{P} 01275$ & Pro-glucagon \\
\hline 2645 & GCK & P35557 & Hexokinase- 4 \\
\hline 2690 & GHR & P10912 & Growth hormone receptor \\
\hline 3156 & HMGCR & P04035 & 3-Hydroxy-3-methylglutaryl-Coenzyme A reductase \\
\hline 3162 & HMOX1 & P09601 & Heme oxygenase 1 \\
\hline 3290 & HSD11B1 & P28845 & Corticosteroid 11-beta-dehydrogenase isozyme 1 \\
\hline 3553 & IL1B & P01584 & Interleukin-1 beta \\
\hline 3569 & IL6 & P05231 & Interleukin-6 \\
\hline 3630 & INS & P01308 & Insulin \\
\hline 3643 & INSR & P06213 & Insulin receptor \\
\hline 3718 & JAK3 & P52333 & Tyrosine-protein kinase JAK3 \\
\hline 3949 & LDLR & $\mathrm{P} 01130$ & Low-density lipoprotein receptor \\
\hline 5595 & MAPK3 & P27361 & Mitogen-activated protein kinase 3 \\
\hline 8972 & MGAM & $\mathrm{O} 43451$ & Maltase-glucoamylase, intestinal \\
\hline 4318 & MMP9 & P14780 & Matrix metalloproteinase- 9 \\
\hline 4552 & MTRR & Q9UBK8 & Methionine synthase reductase \\
\hline 4846 & NOS3 & P29474 & Nitric oxide synthase, endothelial \\
\hline 7376 & $\mathrm{NR} 1 \mathrm{H} 2$ & P55055 & Oxysterols receptor LXR-beta \\
\hline 10062 & NR1H3 & Q13133 & Oxysterols receptor LXR-alpha \\
\hline 9971 & $\mathrm{NR} 1 \mathrm{H} 4$ & Q96RI1 & Bile acid receptor \\
\hline 2908 & NR3C1 & P04150 & Glucocorticoid receptor \\
\hline 5327 & PLAT & P00750 & Tissue-type plasminogen activator \\
\hline 5444 & PON1 & P27169 & Serum paraoxonase/arylesterase 1 \\
\hline 5465 & PPARA & Q07869 & Peroxisome proliferator-activated receptor alpha \\
\hline 5467 & PPARD & Q03181 & Peroxisome proliferator-activated receptor delta \\
\hline 5468 & PPARG & P37231 & Peroxisome proliferator-activated receptor gamma \\
\hline 5617 & PRL & $\mathrm{P} 01236$ & Prolactin \\
\hline 5747 & PTK2 & Q05397 & Focal adhesion kinase 1 \\
\hline 6256 & RXRA & P19793 & Retinoic acid receptor RXR-alpha \\
\hline 6401 & SELE & P16581 & E-selectin \\
\hline 6403 & SELP & P16109 & P-selectin \\
\hline 6462 & SHBG & P04278 & Sex hormone-binding globulin \\
\hline 6647 & SOD1 & P00441 & Superoxide dismutase $[\mathrm{Cu}-\mathrm{Zn}]$ \\
\hline 6652 & SORD & Q00796 & Sorbitol dehydrogenase \\
\hline 6721 & SREBF2 & Q12772 & Sterol regulatory element-binding protein 2 \\
\hline
\end{tabular}


TABLE 2: Continued.

\begin{tabular}{lccc}
\hline Entrez ID & Gene symbol & Uniprot ID & Protein name \\
\hline 7077 & TIMP2 & P16035 & Metalloproteinase inhibitor 2 \\
7099 & TLR4 & O00206 & Toll-like receptor 4 \\
7124 & TNF & P01375 & Tumor necrosis factor \\
7412 & VCAM1 & P19320 & Vascular cell adhesion protein 1 \\
7422 & VEGFA & P15692 & Vascular endothelial growth factor A \\
\hline
\end{tabular}

$\mathrm{CC}=0.4099)$. The top three targets with the highest degree value in the network were AKR1B1 (degree $=13$, $\mathrm{BC}=0.0219, \quad$ and $\mathrm{CC}=0.5000), \quad \mathrm{EGFR} \quad($ degree $=13$, $\mathrm{BC}=0.0202$, and $\mathrm{CC}=0.5000)$, and ESR1 (degree $=13$, $\mathrm{BC}=0.0188$, and $\mathrm{CC}=0.5000)$, suggesting that they are crucial genes involved in the effect of Gegen on targeting T2DM with hyperlipidemia, and TNF, NOS3, MAPK3, PPAR- $\gamma$, PPAR- $\alpha$, ESR2, CYP19A1, INS, IL6, and SORD are also relatively important targets (see Supplementary Material 4 for more topological parameters).

Moreover, signaling pathways are an essential part of system pharmacology and associate receptor-ligand interactions with pharmacodynamic outputs. Sixty of all targets were mapped to 96 KEGG pathways (see Supplementary Material 3 for more details). Twelve of the top 20 pathways, according to the adjusted $P$ value, are closely related to metabolic disorders. These pathways are the PI3K-Akt signaling pathway (degree $=14$ ), AGE-RAGE signaling pathway (degree $=11$ ), fluid shear stress and atherosclerosis (degree $=11)$, endocrine resistance $($ degree $=9)$, HIF-1 signaling pathway (degree $=9)$, PPAR signaling pathway (degree $=8)$, insulin resistance (degree $=8)$, TNF signaling pathway $($ degree $=8)$, nonalcoholic fatty liver disease (degree $=8)$, prolactin signaling pathway $($ degree $=6)$, type II diabetes mellitus (degree $=5$ ), and ovarian steroidogenesis (degree $=5$ ), and much more information is available in Supplementary Material 4.

\section{Discussion}

Currently, the increase in the global prevalence of metabolic diseases represented by T2DM and hyperlipidemia has become an urgent public health problem to solve. T2DM is recognized as a chronic metabolic disorder that affects carbohydrate, lipid, and protein metabolism. A high prevalence of dyslipidemia is a typical feature of T2DM, which has been confirmed in clinical trials $[36,37]$. In turn, hyperlipidemia leading to insulin resistance was realized early and strongly proven by clinical trials [38]. Therefore, the two disorders usually coexist because of the complicated connections between them. Undoubtedly, these diseases have imposed tremendous pressure on international public health, especially the prevention and treatment of cardiovascular complications.

As a medication therapy with a long history, traditional Chinese medicine is known for its multitarget synergistic efficacy, based on which homotherapy for heteropathy is put into practice. However, the elucidation of the multitarget effects of TCM is extremely challenging. With the rapid development of computer technology, bioinformatics, proteomics, and network pharmacology have been successfully applied to discover the active ingredients of Chinese herbs and their pharmacological mechanisms. Gegen, a clinically popular Chinese herb, has been prescribed for DM (also known as Xiaoke in TCM) and hyperlipidemia (similar to blood stasis or phlegm retention syndrome in TCM) for many years. In addition, Gegen has the advantage of longterm use with security due to its edibility. Based on these results, Gegen is one of the optimized candidates for diabetic and hyperlipidemic drug development.

In the present study, we screened 12 active components of Gegen and 65 hub targets using network pharmacology. The PPI analysis shows that INS, encoding the insulin protein that plays a vital role in regulating carbohydrate and lipid metabolism, is the gene with the highest degree. Thus, glucose and lipid metabolic disorders are the key to the occurrence of the two diseases discussed, and one of the main benefits of Gegen is to ameliorate this disorder. In addition, cholesterol metabolism, inflammation, and oxidative stress may also be potential mechanisms by which Gegen regulates metabolic disorders. Moreover, module 1 extracted from the PPI network suggests that the excessive accumulation of oxides (GO: 1901700), such as reactive oxygen species, may be one of the common pathological features of metabolic dysfunction, consistent with previous studies that are well summarized in the literature [39]. Module 2 implies that these targets have a significant role in regulating cholesterol metabolism, especially the speed or degree of cholesterol storage (GO: 0010885).

The GO enrichment analysis further revealed that Gegen may regulate lipid metabolism, especially cholesterol metabolism, steroid metabolism, response to nutrient levels, and the inflammatory response through ligand binding, signal transduction, and fatty acid binding in blood, vesicle lumen, caveolae, cell membranes, cytoplasm, and other sites to improve metabolic disorders and exert anti-inflammatory effects. Interestingly, a large number of statistically significant BP terms, such as response to insulin, regulation of insulin secretion, insulin secretion, cellular response to insulin stimulus, glucose metabolic process, glucose homeostasis, and response to glucose, were not at the top of the list (see Supplementary Material 3 for more details). We inferred that the regulatory effect of Gegen on lipids may be more definite than its effect on glucose control from the perspective of evidence-based medicine because the GO annotation and obsoleting are completely based on the latest evidence [40]. Moreover, the benefits of Gegen in treating T2DM and hyperlipidemia are not limited to lowering serum lipid and glucose profiles directly because it also resolves inflammation during the progression of these diseases. 


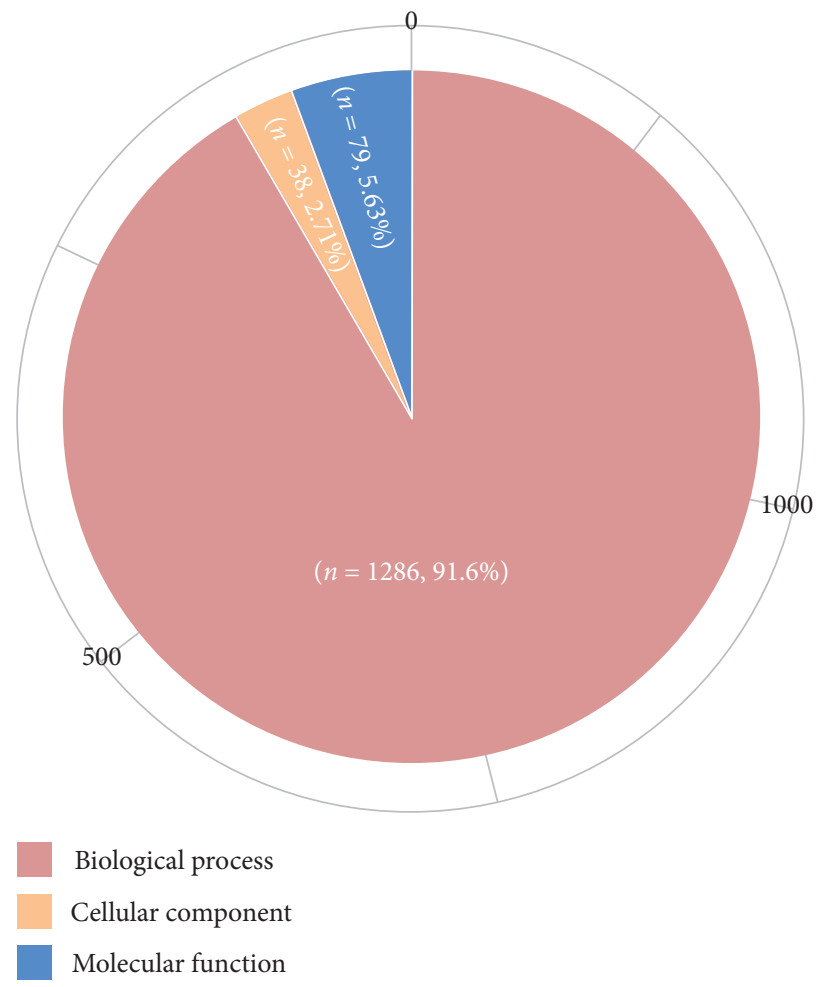

(a)

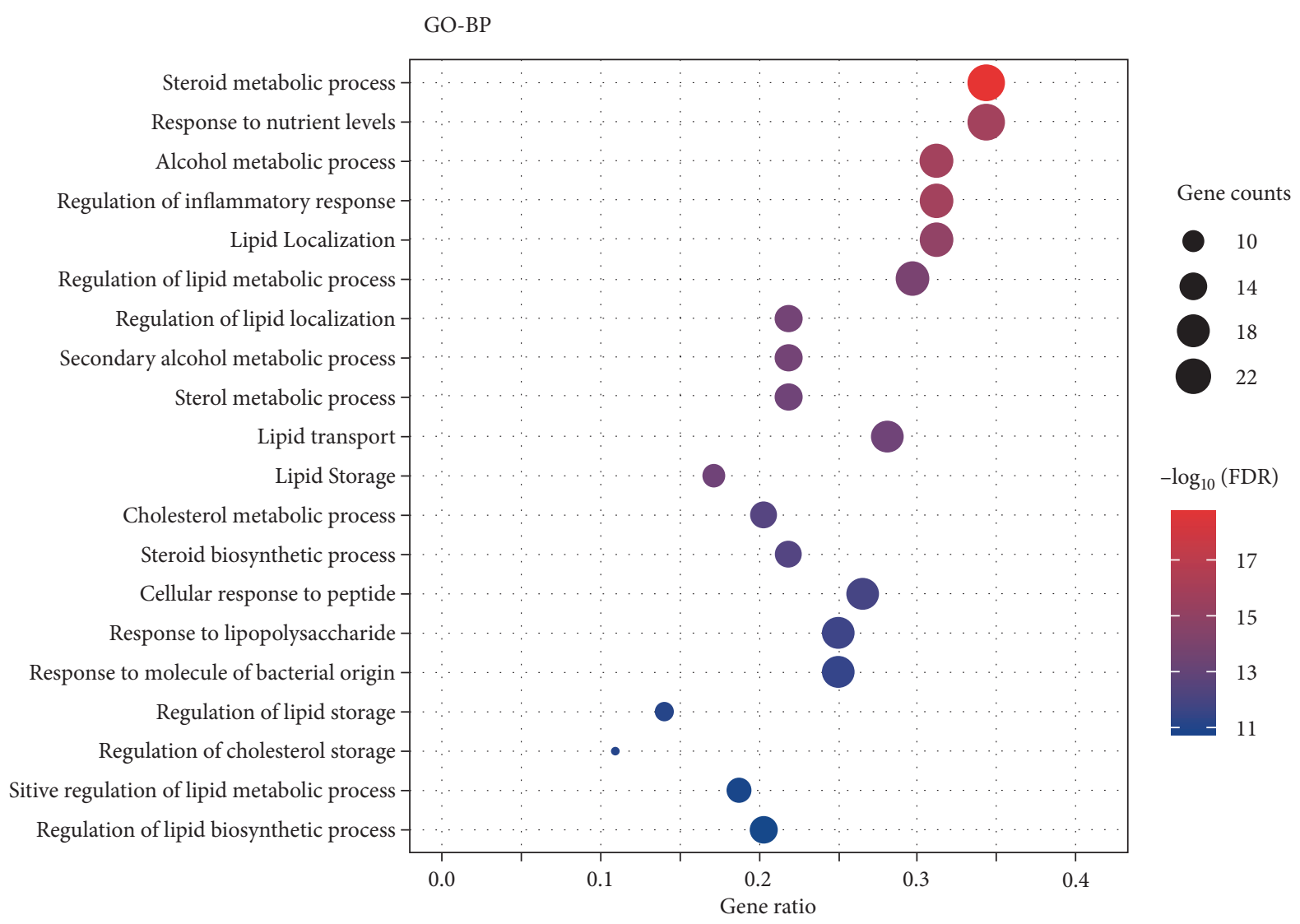

(b)

Figure 2: Continued. 
GO-CC

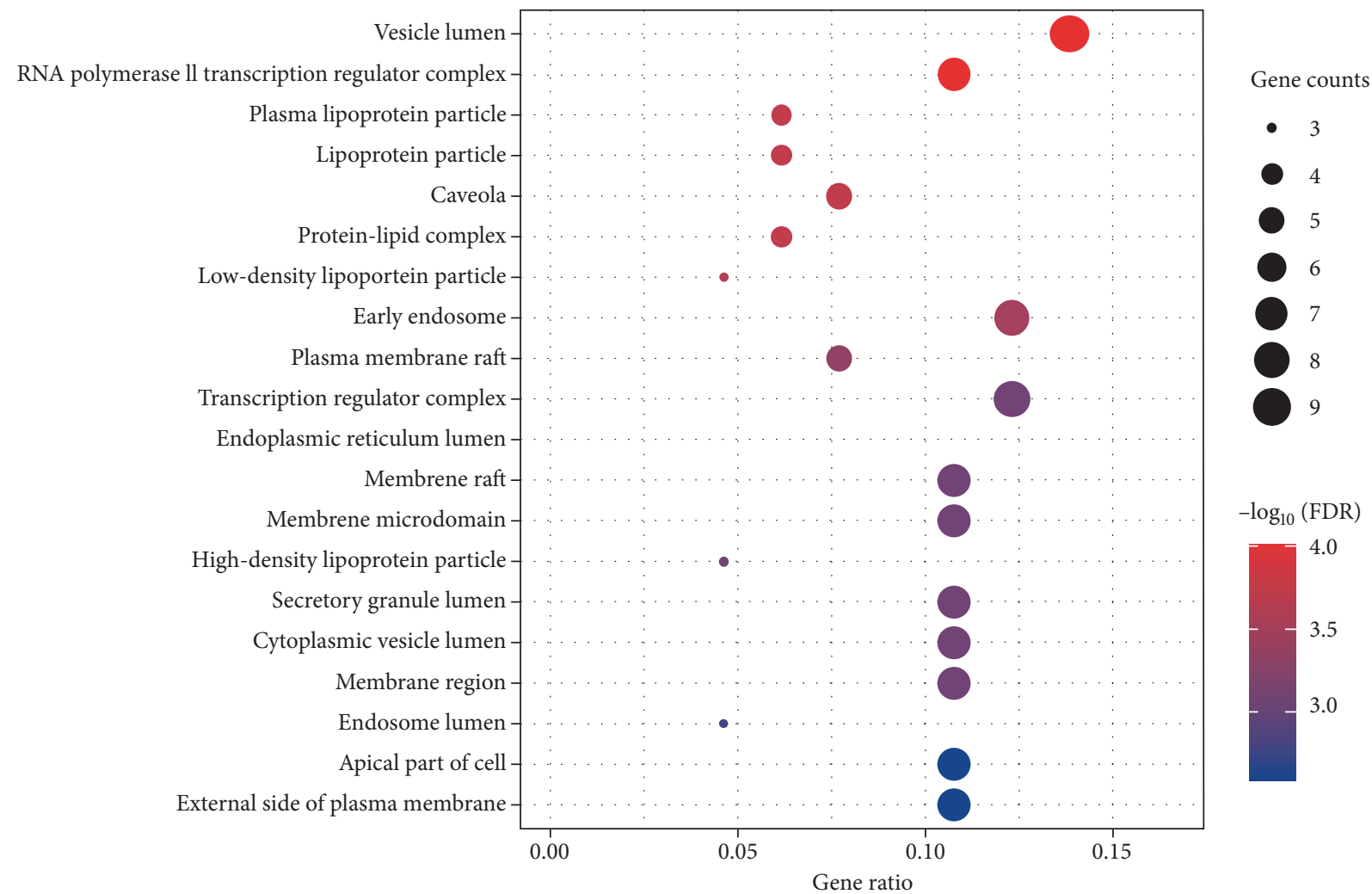

(c)

GO-MF

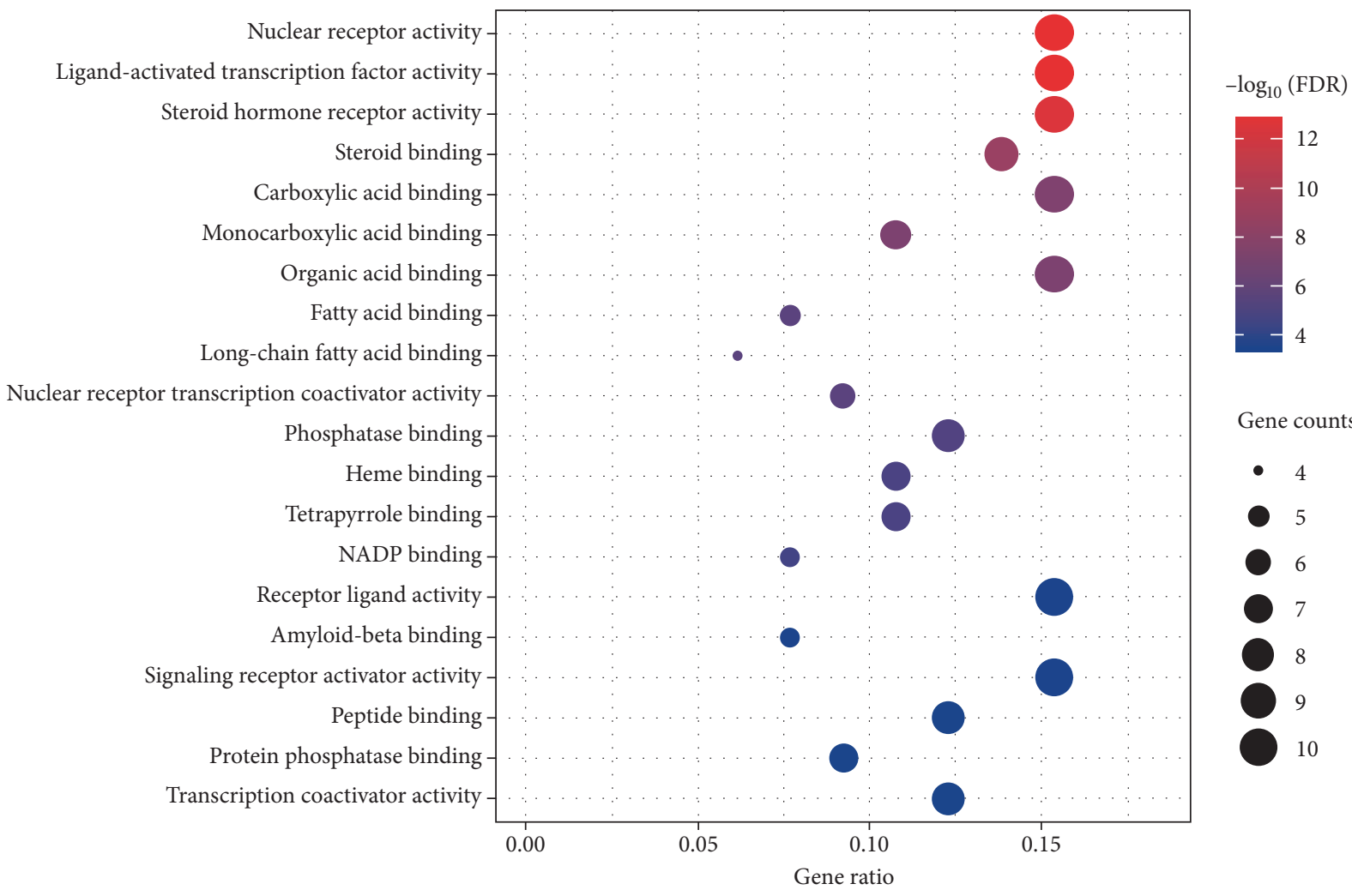

(d)

FIGURE 2: GO enrichment analysis of the 65 common targets of Gegen, T2DM, and hyperlipidemia. (a) The ratio of the GO terms. Biological processes (red), cellular components (yellow), and molecular functions (blue) accounted for 91.60\%, 2.78\%, and 5.63\%, respectively. (b-d) Bubble plots of the top $20 \mathrm{GO}$ terms for biological processes, cellular components, and molecular functions. Abbreviations: BP, biological process; CC, cellular component; MF, molecular function; FDR, false discovery rate, namely, the adjusted $P$ value. 


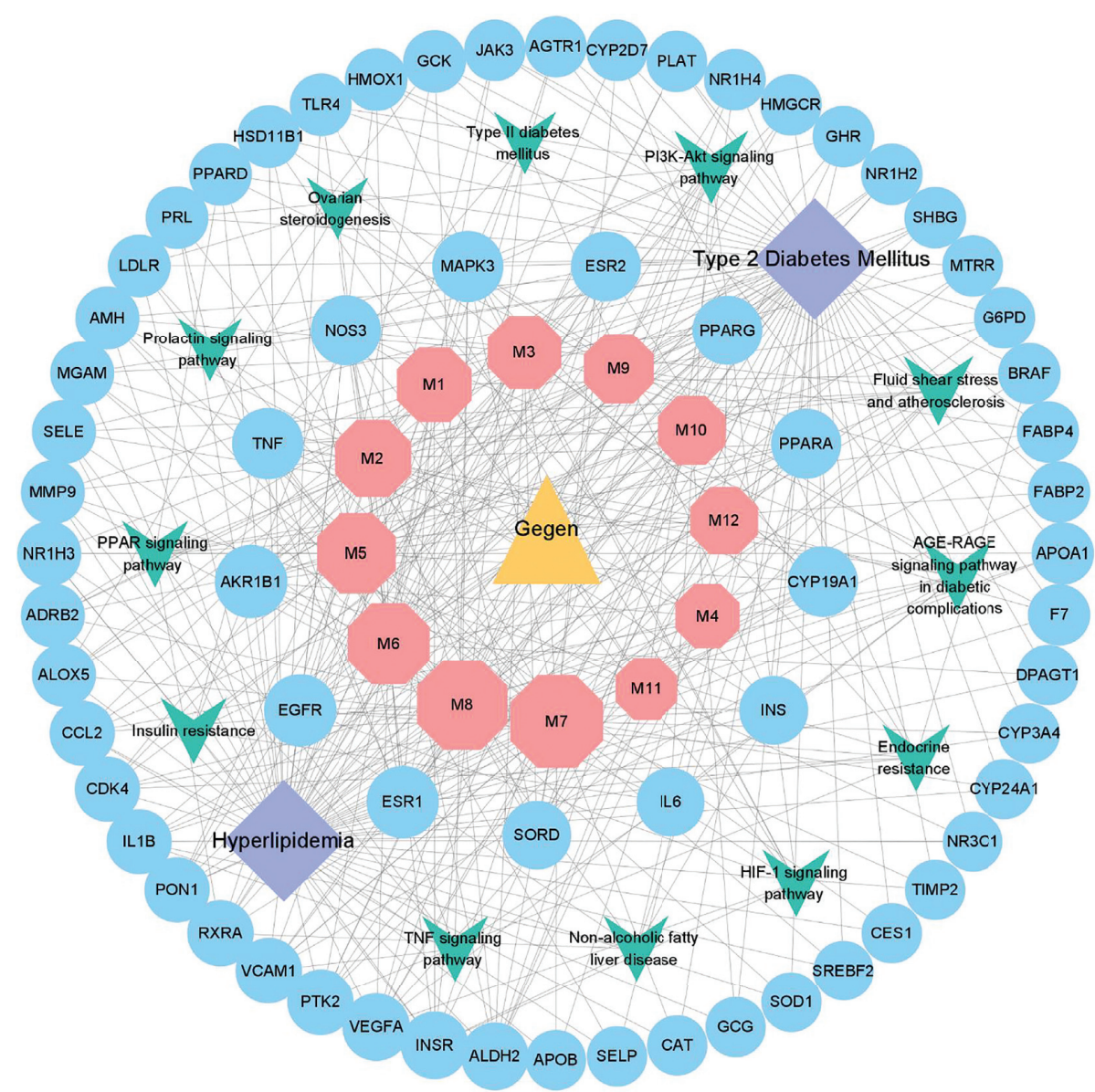

FIGURE 3: Compound-target-pathway network. The yellow triangle represents the herb, the pink hexagons represent the active ingredients of Gegen, the blue circles represent the common targets between the compounds and the diseases, the purple diamonds represent the diseases, and the green $\mathrm{V}$ shapes represent the pathways. Edges represent the interactions among ingredients, targets, and pathways. The greater the number of edges connected to the same node, the larger the size of the node.

By constructing a three-layer network of compounds, targets, and pathways, we obtained direct insights into the complex interactions among them. We found that these targets are widely involved in insulin resistance and sensitization, glucose and lipid metabolism, inflammation, and diabetes complications. For example, AKR1B1 and SORD have been proven to be associated with the occurrence of complications such as diabetic neuropathy [41, 42]. INS and PPARs are undoubtedly some of the most representative genes regulating metabolism. Insulin plays a vital role in the regulation of saccharides and lipid metabolism. PPAR- $\alpha$ is a key regulator of lipid metabolism, such as clearing circulating or cellular lipids. PPAR- $\gamma$ promotes adipocyte differentiation and increases glucose uptake, processes that are essential in the prevention of obesity and the treatment of type 2 diabetes [43].

In addition, EGFR, a gene traditionally considered to regulate cell proliferation and fibrosis, has been shown to be involved in maintaining metabolic homeostasis. Scheving et al. were the first to show that mice with gain-of-function point mutations in the kinase domain of EGFR display elevated plasma and hepatic cholesterol and plasma LDL levels, proving the role of EGFR in regulating lipid metabolism at the basal level [44]. Since then, an increasing number of researchers have verified the role of EGFR in regulating metabolism. Fang et al. observed that EGFR inhibitors were able to reduce inflammation, oxidative stress, fibrosis, and apoptosis in palmitic acid-treated NRK-52E cells and kidneys of high-fat diet-fed mice and improved serum lipid levels and body weight [45]. Estrogen is very important in the metabolic regulation of the whole body, and estrogen receptor (ER) is highly involved in estrogenmediated modulation of substrate metabolism. ESR1 is critical for the maintenance of whole-body insulin action and protection against tissue inflammation. According to a previous study, ESR1-knockout female mice exhibited significant weight gain, obviously higher fasting blood glucose and lipid levels, elevated levels of circulating and tissue inflammatory markers (PAI-1, MAPK8, and TNF), increased muscular lipid accumulation, impaired glucose tolerance, and insulin resistance, even when they were fed a normal chow diet [46]. In terms of clinical research, some studies have shown that ESR1 and ESR2 gene polymorphisms are associated with lipid levels and insulin sensitivity in adults, despite racial and sex variability [47-49]. TNF- $\alpha$ is one of the most important proinflammatory mediators and a 
key factor in insulin resistance, the evidence for which was well summarized in a classic review [50]. In addition, in healthy humans, TNF- $\alpha$ was proven to inhibit whole-body insulin-mediated glucose uptake and signal transduction by suppressing peripheral insulin-stimulated glucose uptake [51]. NOS3 is crucial for the control of arterial pressure and glucose and lipid homeostasis. NOS3-/- mice appeared hypertensive and presented fasting hyperinsulinemia, hyperlipidemia, and lower insulin-stimulated glucose uptake than wild-type mice [52]. Apart from indirectly reducing the risk of cardiovascular and cerebrovascular adverse events by regulating glucose and lipid metabolism, NOS3 also exerts direct effects on vascular protection by synthesizing NO, which is reported to function as an anti-inflammatory agent and antioxidant, including maintaining vascular homeostasis, maintaining the dilation of the vasculature, protecting the intima, and preventing smooth muscle proliferation $[53,54]$. Last but not least, approaches targeting MAPK3 (also known as extracellular signal-regulated kinase 1, ERK1) partially protect obese mice from insulin resistance and hepatic steatosis by decreasing adipose tissue inflammation and by increasing muscle glucose uptake [55].

Consistently, the major bioactive components of Gegen have been widely shown to regulate these targets. Thus, the rich isoflavones in Gegen are the dominant active ingredients responsible for the antidiabetes and antihyperlipidemia effects, including daidzein and genistein that are commonly found in soybeans, as well as puerarin, formononetin, and $3^{\prime}$-methoxydaidzein. Isoflavones are phytoestrogens with potent estrogenic activity that have structural similarity with the human female hormone 17- $\beta$-estradiol. Hence, isoflavones bind to both alpha and beta estrogen receptors and mimic the action of estrogens on target organs. In addition to estrogen-like and/or antiestrogen activity, numerous studies have claimed the functions of genistein and daidzein in the maintenance of metabolic homeostasis and anti-inflammatory and antioxidant activities, thereby exerting many benefits of chemoprevention of metabolic syndrome (MS), obesity, and cardiovascular disease, as well as in relieving postmenopausal symptoms [56-59]. In terms of metabolic regulation, Zucker rats and RAW 264.7 cells treated with a protein mixture or extract of genistein and/or daidzein exhibited antidiabetic effects similar to PPAR agonists, with improved lipid metabolism and activated PPAR receptors [60]. Clinically, a meta-analysis of seventeen randomized controlled trials showed that soy isoflavones significantly improve glucose metabolism in menopausal women [61]. With respect to inhibiting the inflammatory response and oxidative stress, genistein was reported to ameliorate fatty liver in insulinresistant rats by activating the antioxidant profile, decreasing IL6 and TNF- $\alpha$ concentrations and preventing oxidative damage [62]. In addition, apoptosis and proliferation inhibition in human umbilical vein endothelial cells incubated with hydrogen peroxide and high glucose are prevented by genistein and daidzein through the regulation of ESR2 and Bcl-2/Bax expression and modulation of cell survival-related signaling pathways, such as the PI3K pathway [63].

As the most abundant secondary metabolite, puerarin is a unique isoflavone of Gegen. Due to its multiple pharmacological functions, such as vasodilation, cardioprotection, and antioxidant and anti-inflammatory effects, along with the attenuation of insulin resistance, puerarin has been widely used to treat cardiovascular and cerebrovascular diseases, diabetes, and diabetic complications [64], as proven in vivo and in vitro. Puerarin exerts positive hypoglycemic and hypolipidemic roles on mice with diabetes induced by streptozotocin by increasing insulin expression and maintaining metabolic homoeostasis, and it exerts a regulatory effect on lipid accumulation in oleic acidtreated HepG2 cells by decreasing lipogenesis and increasing antioxidant activity, both indicating that puerarin extract has therapeutic benefits in the treatment of glucose- and lipid-related metabolic disorders $[65,66]$. Puerarin was able to dose-dependently reduce the phosphorylation of ERK, the expression of TNF- $\alpha$ and NOS3, and the release of TNF- $\alpha$ and NO to inhibit inflammatory signaling in RAW264.7 macrophages treated with high concentrations of free fatty acids, which are often increased in patients with T2DM and MS [67].

$\beta$-Sitosterol, a prevalent plant cholesterol derivative (phytosterol) known as the "key of life", is another pivotal active ingredient in Gegen. As a natural PPAR- $\gamma$ agonist, $\beta$-sitosterol could be used as a potential therapeutic phytomedicine for the management of metabolic disorders, and overwhelming evidence has been obtained from basic experiments. For example, Gumede et al. discovered that $\beta$-sitosterol prevents high-fructose diet-induced metabolic dysfunction in female rats, including visceral obesity, hypertriglyceridemia, and hypoadiponectinemia [68]. Another in vivo study showed that the administration of $\beta$-sitosterol significantly increased the levels of insulin, hemoglobin, and the PPAR- $\gamma$ and GLUT4 proteins, alleviated insulin resistance, and decreased the plasma glucose levels in high-fat diet- and streptozotocin-induced diabetic rats [69]. In addition, a clinical cohort study revealed rather specific negative correlations between the serum sitosterol level and the serum IL6 and TNF- $\alpha$ levels in both subjects with and without diabetes, suggesting the anti-inflammatory potential of $\beta$-sitosterol [70].

Interacting targets form signaling pathways that may reveal the mechanism of a disease. The PI3K-Akt signaling pathway, PPAR signaling pathway, and insulin resistance play pivotal roles in insulin secretion, glucose uptake, and lipid metabolism to maintain glucose and lipid homeostasis $[71,72]$. The AGE-RAGE signaling pathway has been proven to exert critical effects on the occurrence and development of diabetic complications, especially vascular complications, and the evidence based on a large number of studies was reviewed elsewhere [73]. Fluid shear stress and atherosclerosis, the HIF-1 signaling pathway, and the TNF signaling pathway are primarily involved in the secretion of proinflammatory factors, matrix degradation, angiogenesis, regulation of vascular tone, leucocyte recruitment, and cell adhesion, which are a series of inflammation-related events that eventually lead to vasculopathy, such as atherosclerosis.

In summary, the main active ingredients of Gegen are isoflavones, such as daidzein, genistein, and puerarin, as well as $\beta$-sitosterol, a natural triterpenoid. To some extent, these 
components have consistent multitarget effects. Briefly, the efficacy of Gegen is mainly attributed to regulating insulin secretion and sensitivity, glucose metabolism, and fatty acid and cholesterol synthesis and decomposition to maintain metabolic homeostasis (INS, PPAR- $\gamma$, PPAR- $\alpha$, and EGFR). On the other hand and more importantly, it also regulates the expression of proteins that mediate complications and risk factors for diabetes and hyperlipidemia, including diabetic cataracts, diabetic retinopathy, diabetic neuropathies, inflammation, atherosclerosis, liver steatosis, and obesity (AKR1B1, SORD, TNF, NOS3, IL6, EGFR, ESR1, and ESR2), to alleviate pathological progression and improve the prognosis. Thus, we speculated that the abundant bioactive ingredients of Gegen may work simultaneously on multiple targets in the PI3K-Akt signaling pathway, AGE-RAGE signaling pathway, fluid shear stress, and atherosclerosis to exert a synergistic therapeutic effect on T2DM and hyperlipidemia, which not only regulates glucose and lipid metabolism directly but also prevents and improves complications secondary to glucose and lipid metabolic disorders. Of course, this study has some limitations. We did not perform in vivo or in vitro experiments to validate the results. We would be willing to supplement the research in the future if the conditions are available and even try to design a clinical trial for verification.

\section{Conclusions}

Taken together, we have discovered that isoflavones and $\beta$-sitosterol are the critical active components of Gegen responsible for its antidiabetic and antihyperlipidemia effects, which involve multiple mechanisms, such as ameliorating insulin resistance, increasing glucose uptake, promoting adipocyte differentiation, reducing lipid accumulation, and resisting inflammation and oxidative stress. These results are consistent with the main findings of previous studies, providing valuable information for the further development of Gegen. Specifically, Gegen or its bioactive extract has the potential to be applied to the treatment of T2DM and hyperlipidemia independently as a new medicine. After all, Gegen has been used only in the form of a compound prescription (a prescription consisting of 2 or more different Chinese medicines) for clinical treatment to date. Compared with Chinese herbal compounds, the mechanism of action of single Chinese herbs and monomeric compounds is easier to explain and apply in the clinic. Overall, Gegen is a safe, effective, and promising natural phytomedicine for the treatment of type 2 diabetes accompanied by hyperlipidemia with the advantages of multiple ingredients, targets, biological processes, and pathways.

\section{Data Availability}

The data used to support the findings of this study are included within the supplementary information files.

\section{Conflicts of Interest}

The authors declare that there are no conflicts of interest regarding the publication of this paper.

\section{Authors' Contributions}

Guozhen Yuan and Shuai Shi contributed equally to this work.

\section{Acknowledgments}

The authors thank Mr. Wang Chuang, a Java engineer from State Grid (Beijing, China), for his guidance and help on software and scripts.

\section{Supplementary Materials}

Supplementary materials include the following. Supplementary Material 1: active ingredients and targets of Gegen. Supplementary Material 2: targets of type 2 diabetes and hyperlipidemia. Supplementary Material 3: results of GO and KEGG enrichment. Supplementary Material 4: topological parameters of the compound-target-pathway network. (Supplementary Materials)

\section{References}

[1] N. H. Cho, J. E. Shaw, S. Karuranga et al., "IDF diabetes atlas: global estimates of diabetes prevalence for 2017 and projections for 2045," Diabetes Research and Clinical Practice, vol. 138, pp. 271-281, 2018.

[2] International Diabetes Federation, IDF Diabetes Atlas Ninth Edition, International Diabetes Federation, Brussels, Belgium, 2019, https://diabetesatlas.org/en/resources/.

[3] P. Libby, J. E. Buring, L. Badimon et al., "Atherosclerosis," Nature Reviews Disease Prime, vol. 5, no. 1, p. 56, 2019.

[4] Ž. Reiner, "Hypertriglyceridaemia and risk of coronary artery disease," Nature Reviews Cardiology, vol. 14, no. 7, pp. 401-411, 2017.

[5] A. M. Navar-Boggan, E. D. Peterson, R. B. D’Agostino, B. Neely, A. D. Sniderman, and M. J. Pencina, "Hyperlipidemia in early adulthood increases long-term risk of coronary heart disease," Circulation, vol. 131, no. 5, pp. 451-458, 2015.

[6] M. S. Mouradian, S. R. Majumdar, A. Senthilselvan, K. Khan, and A. Shuaib, "How well are hypertension, hyperlipidemia, diabetes, and smoking managed after a stroke or transient ischemic attack?" Stroke, vol. 33, no. 6, pp. 1656-1659, 2002.

[7] H. Tunstall-Pedoe, R. Chen, and P Kramarz, "Prevalence of individuals with both raised blood pressure and raised cholesterol in WHO MONICA project population surveys 1989-97," Pharmacoepidemiology and Drug Safety, vol. 13, no. 1, pp. S307-S308, 2004.

[8] J. Q. Xu, K. D. Kochanek, S. L. Murphy, and B Tejada-Vera, "Deaths: final data for 2007," National Vital Statistics Reports, vol. 58, no. 19, pp. 1-136, 2010.

[9] National Health and Family Planning Commission Disease Prevention and Control Bureau, 2015 Report on Chinese Resident's Chronic Disease and Nutrition, People's Medica Publishing House, Beijing, China, 1st edition, 2015.

[10] L. Brunetti and E. R. Hermes-Desantis, "The role of colesevelam hydrochloride in hypercholesterolemia and type 2 diabetes mellitus," Annals of Pharmacotherapy, vol. 44, no. 78, pp. 1196-1206, 2010.

[11] S. S. Virani, A. Alonso, E. J. Benjamin et al., "Heart disease and stroke statistics-2020 update: a report from the American Heart Association," Circulation, vol. 141, no. 9, pp. e139-e596, 2020. 
[12] P. K. Battiprolu, T. G. Gillette, Z. V. Wang, S. Lavandero, and J. A. Hill, "Diabetic cardiomyopathy: mechanisms and therapeutic targets," Drug Discovery Today: Disease Mechanisms, vol. 7, no. 2, pp. e135-e143, 2010.

[13] G. Y. Chen, L. Li, F. Dai, X. J. Li, X. X. Xu, and J. G Fan, "Prevalence of and risk factors for type 2 diabetes mellitus in hyperlipidemia in China," Medical Science Monitor: International Medical Journal of Experimental and Clinical Research, vol. 21, pp. 2476-2484, 2015.

[14] V. G. Athyros, M. Doumas, K. P. Imprialos et al., "Diabetes and lipid metabolism," Hormones, vol. 17, no. 1, pp. 61-67, 2018.

[15] S. Higuchi, M. C. Izquierdo, and R. A. Haeusler, "Unexplained reciprocal regulation of diabetes and lipoproteins," Current Opinion in Lipidology, vol. 29, no. 3, pp. 186-193, 2018.

[16] M. Gupta, R. Tummala, R. K. Ghosh et al., "An update on pharmacotherapies in diabetic dyslipidemia," Progress in Cardiovascular Diseases, vol. 62, no. 4, pp. 334-341, 2019.

[17] M. Paquette, S. Bernard, I. Ruel, D. W. Blank, J. Genest, and A. Baass, "Diabetes is associated with an increased risk of cardiovascular disease in patients with familial hypercholesterolemia," Journal of Clinical Lipidology, vol. 13, no. 1, pp. 123-128, 2019.

[18] Y. Cho, E. Choe, Y.-h. Lee et al., "Risk of diabetes in patients treated with HMG-CoA reductase inhibitors," Metabolism, vol. 64, no. 4, pp. 482-488, 2015.

[19] D. M. Nathan, P. H. Bennett, P. H. Bennett et al., "Does diabetes prevention translate into reduced long-term vascular complications of diabetes?" Diabetologia, vol. 62, no. 8, pp. 1319-1328, 2019.

[20] H. Lv, J. Liu, C. Zhen et al., "Magnetic fields as a potential therapy for diabetic wounds based on animal experiments and clinical trials," Cell Proliferation, vol. 54, no. 3, Article ID e12982, 2021.

[21] M. G. Savelieff, B. C. Callaghan, and E. L. Feldman, "The emerging role of dyslipidemia in diabetic microvascular complications," Current Opinion in Endocrinology \& Diabetes and Obesity, vol. 27, no. 2, pp. 115-123, 2020.

[22] J. Xu, F. Lian, L. Zhao et al., "Structural modulation of gut microbiota during alleviation of type 2 diabetes with a Chinese herbal formula," The ISME Journal, vol. 9, no. 3, pp. 552-562, 2015.

[23] T. Kwok, P. C. Leung, C. Lam et al., “A randomized placebo controlled trial of an innovative herbal formula in the prevention of atherosclerosis in postmenopausal women with borderline hypercholesterolemia," Complementary Therapies in Medicine, vol. 22, no. 3, pp. 473-480, 2014.

[24] C.-M. Liu, J.-Q. Ma, and Y.-Z. Sun, "Protective role of puerarin on lead-induced alterations of the hepatic glutathione antioxidant system and hyperlipidemia in rats," Food and Chemical Toxicology, vol. 49, no. 12, pp. 3119-3127, 2011.

[25] N. Thomford, D. Senthebane, A. Rowe et al., "Natural products for drug discovery in the 21st century: innovations for novel drug discovery," International Journal of Molecular Sciences, vol. 19, no. 6, p. 1578, 2018.

[26] M. H. Baig, K. Ahmad, S. Roy et al., "Computer aided drug design: success and limitations," Current Pharmaceutical Design, vol. 22, no. 5, pp. 572-581, 2016.

[27] P. Shannon, A. Markiel, O. Ozier et al., "Cytoscape: a software environment for integrated models of biomolecular interaction networks," Genome Research, vol. 13, no. 11, pp. 2498-2504, 2003.
[28] G. D. Bader and C. W. Hogue, "An automated method for finding molecular complexes in large protein interaction networks," BMC Bioinformatics, vol. 4, no. 1, p. 2, 2003.

[29] M. Ashburner, C. A. Ball, J. A. Blake et al., "Gene ontology: tool for the unification of biology. The gene ontology consortium," Nature Genetics, vol. 25, no. 1, pp. 25-29, 2000.

[30] D. Vella, S. Marini, F. Vitali, D. Di Silvestre, G. Mauri, and R. Bellazzi, "MTGO: PPI network analysis via topological and functional module identification," Scientific Reports, vol. 8, no. 1, p. 5499, 2018.

[31] M. Kanehisa, S. Goto, M. Furumichi, M. Tanabe, and M. Hirakawa, "KEGG for representation and analysis of molecular networks involving diseases and drugs," Nucleic Acids Research, vol. 38, no. 1, pp. D355-D360, 2010.

[32] G. Yu, L.-G. Wang, Y. Han, and Q.-Y. He, "clusterProfiler: an $\mathrm{R}$ package for comparing biological themes among gene clusters," OMICS: A Journal of Integrative Biology, vol. 16, no. 5, pp. 284-287, 2012.

[33] M. Carlson, "org.Hs.eg.db: Genome wide annotation for human. R package version 3.11.4.," 2020, http://bioconductor. org/news/bioc_3_11_release/.

[34] R. Ihaka and R. Gentleman, "R: a language for data analysis and graphics," Journal of Computational and Graphical Statistics, vol. 5, no. 3, pp. 299-314, 1996.

[35] Y. Assenov, F. Ramírez, S.-E. Schelhorn, T. Lengauer, and M. Albrecht, "Computing topological parameters of biological networks," Bioinformatics, vol. 24, no. 2, pp. 282-284, 2008.

[36] B. Pantoja-Torres, C. J. Toro-Huamanchumo, D. UrrunagaPastor et al., "High triglycerides to HDL-cholesterol ratio is associated with insulin resistance in normal-weight healthy adults," Diabetes \& Metabolic Syndrome: Clinical Research \& Reviews, vol. 13, no. 1, pp. 382-388, 2019.

[37] N. Wang, J. Cheng, Z. Ning et al., "Type 2 diabetes and adiposity induce different lipid profile disorders: a mendelian randomization analysis," The Journal of Clinical Endocrinology \& Metabolism, vol. 103, no. 5, pp. 2016-2025, 2018.

[38] T. Han, Y. Cheng, S. Tian et al., "Changes in triglycerides and high-density lipoprotein cholesterol may precede peripheral insulin resistance, with 2 -h insulin partially mediating this unidirectional relationship: a prospective cohort study," Cardiovascular Diabetology, vol. 15, no. 1, p. 154, 2016.

[39] S. J. Forrester, D. S. Kikuchi, M. S. Hernandes, Q. Xu, and K. K. Griendling, "Reactive oxygen species in metabolic and inflammatory signaling," Circulation Research, vol. 122, no. 6, pp. 877-902, 2018.

[40] The Gene Ontology Consortium, "The gene ontology resource: 20 years and still going strong," Nucleic Acids Research, vol. 47, no. D1, pp. D330-D338, 2019.

[41] S. Abhary, A. W. Hewitt, K. P. Burdon, and J. E. Craig, "A systematic meta-analysis of genetic association studies for diabetic retinopathy," Diabetes, vol. 58, no. 9, pp. 2137-2147, 2009.

[42] A. Cortese, Y. Zhu, A. P. Rebelo et al., "Biallelic mutations in SORD cause a common and potentially treatable hereditary neuropathy with implications for diabetes," Nature Genetics, vol. 52, no. 5, pp. 473-481, 2020.

[43] X. Shao, M. Wang, X. Wei et al., "Peroxisome proliferatoractivated receptor- $\gamma$ : master regulator of adipogenesis and obesity," Current Stem Cell Research \& Therapy, vol. 11, no. 3, pp. 282-289, 2016.

[44] L. A. Scheving, X. Zhang, O. A. Garcia et al., "Epidermal growth factor receptor plays a role in the regulation of liver and plasma lipid levels in adult male mice," American Journal 
of Physiology-Gastrointestinal and Liver Physiology, vol. 306, no. 5, pp. G370-G381, 2014.

[45] Q. Fang, C. Zou, P. Zhong et al., "EGFR mediates hyperlipidemia-induced renal injury via regulating inflammation and oxidative stress: the detrimental role and mechanism of EGFR activation," Oncotarget, vol. 7, no. 17, pp. 24361-24373, 2016.

[46] V. Ribas, M. T. A. Nguyen, D. C. Henstridge et al., "Impaired oxidative metabolism and inflammation are associated with insulin resistance in ER $\alpha$-deficient mice," American Journal of Physiology-Endocrinology and Metabolism, vol. 298, no. 2, pp. E304-E319, 2010.

[47] Q. Huang, T.-h. Wang, W.-s. Lu et al., "Estrogen receptor alpha gene polymorphism associated with type 2 diabetes mellitus and the serum lipid concentration in Chinese women in Guangzhou," Chinese Medical Journal, vol. 119, no. 21, pp. 1794-1801, 2006.

[48] J. C. Lo, X. Zhao, A. Scuteri, S. Brockwell, and M. R. Sowers, "The association of genetic polymorphisms in sex hormone biosynthesis and action with insulin sensitivity and diabetes mellitus in women at midlife," The American Journal of Medicine, vol. 119, no. 9, pp. S69-S78, 2006.

[49] Z. A. Efstathiadou, C. Sakka, S. A. Polyzos et al., "Associations of estrogen receptor alpha and beta gene polymorphisms with lipid levels and insulin resistance in men," Metabolism, vol. 64, no. 5, pp. 611-617, 2015.

[50] G. S. Hotamisligil, "The role of TNF $\alpha$ and TNF receptors in obesity and insulin resistance," Journal of Internal Medicine, vol. 245 , no. 6 , pp. 621-625, 1999.

[51] P. Plomgaard, K. Bouzakri, R. Krogh-Madsen, B. Mittendorfer, J. R. Zierath, and B. K. Pedersen, "Tumor necrosis factor- induces skeletal muscle insulin resistance in healthy human subjects via inhibition of akt substrate 160 phosphorylation," Diabetes, vol. 54, no. 10, pp. 2939-2945, 2005.

[52] H. Duplain, R. Burcelin, C. Sartori et al., "Insulin resistance, hyperlipidemia, and hypertension in mice lacking endothelial nitric oxide synthase," Circulation, vol. 104, no. 3, pp. 342-345, 2001.

[53] H. Li and U. Förstermann, "Nitric oxide in the pathogenesis of vascular disease," The Journal of Pathology, vol. 190, no. 3, p. 244, Article ID 254, 2000.

[54] D. Tousoulis, A.-M. Kampoli, C. Tentolouris, N. Papageorgiou, and C Stefanadis, "The role of nitric oxide on endothelial function," Current Vascular Pharmacology, vol. 10, no. 1, pp. 4-18, 2012.

[55] J. Jager, V. Corcelle, T. Grémeaux et al., "Deficiency in the extracellular signal-regulated kinase 1 (ERK1) protects leptindeficient mice from insulin resistance without affecting obesity," Diabetologia, vol. 54, no. 1, pp. 180-189, 2011.

[56] L. Křrižová, K. Dadáková, J. Kašparovská, and T Kašparovský, "Isoflavones," Molecules (Basel, Switzerland), vol. 24, no. 6, 2019.

[57] V. Mukund, D. Mukund, V. Sharma, M. Mannarapu, and A. Alam, "Genistein: its role in metabolic diseases and cancer," Critical Reviews in Oncology/Hematology, vol. 119, pp. 13-22, 2017.

[58] D. Das, S. Sarkar, J. Bordoloi, S. B. Wann, J. Kalita, and P. Manna, "Daidzein, its effects on impaired glucose and lipid metabolism and vascular inflammation associated with type 2 diabetes," BioFactors, vol. 44, no. 5, pp. 407-417, 2018.

[59] K. Yamagata, "Soy isoflavones inhibit endothelial cell dysfunction and prevent cardiovascular disease," Journal of Cardiovascular Pharmacology, vol. 74, no. 3, pp. 201-209, 2019.
[60] O. Mezei, W. J. Banz, R. W. Steger, M. R. Peluso, T. A. Winters, and N. Shay, "Soy isoflavones exert antidiabetic and hypolipidemic effects through the PPAR pathways in obese Zucker rats and murine RAW 264.7 cells," The Journal of Nutrition, vol. 133, no. 5, pp. 1238-1243, 2003.

[61] K. Fang, H. Dong, D. Wang, J. Gong, W. Huang, and F. Lu, "Soy isoflavones and glucose metabolism in menopausal women: a systematic review and meta-analysis of randomized controlled trials," Molecular Nutrition \& Food Research, vol. 60, no. 7, pp. 1602-1614, 2016.

[62] S. Mohamed Salih, P. Nallasamy, P. Muniyandi, V. Periyasami, and A. Carani Venkatraman, "Genistein improves liver function and attenuates non-alcoholic fatty liver disease in a rat model of insulin resistance," Journal of Diabetes, vol. 1, no. 4, pp. 278-287, 2009.

[63] S.-Z. Xu, W. Zhong, M. Ghavideldarestani, R. Saurabh, S. W. Lindow, and S. L. Atkin, "Multiple mechanisms of soy isoflavones against oxidative stress-induced endothelium injury," Free Radical Biology and Medicine, vol. 47, no. 2, pp. 167-175, 2009.

[64] Y.-X. Zhou, H. Zhang, and C. Peng, "Puerarin: a review of pharmacological effects," Phytotherapy Research, vol. 28, no. 7, pp. 961-975, 2014.

[65] K. Wu, T. Liang, X. Duan, L. Xu, K. Zhang, and R. Li, “Antidiabetic effects of puerarin, isolated from Pueraria lobata (Willd.), on streptozotocin-diabetogenic mice through promoting insulin expression and ameliorating metabolic function," Food and Chemical Toxicology, vol. 60, pp. 341-347, 2013.

[66] O.-H. Kang, S.-B. Kim, S.-H. Mun et al., "Puerarin ameliorates hepatic steatosis by activating the PPAR $\alpha$ and AMPK signaling pathways in hepatocytes," International Journal of Molecular Medicine, vol. 35, no. 3, pp. 803-809, 2015.

[67] Y.-m. Tu, C.-x. Gong, L. Ding et al., "A high concentration of fatty acids induces TNF- $\alpha$ as well as NO release mediated by the $\mathrm{P} 2 \mathrm{X} 4$ receptor, and the protective effects of puerarin in RAW264.7 cells," Food \& Function, vol. 8, no. 12, pp. 4336-4346, 2017.

[68] N. M. Gumede, B. W. Lembede, R. L. Brooksbank, K. H. Erlwanger, and E. Chivandi, " $\beta$-Sitosterol shows potential to protect against the development of high-fructose diet-induced metabolic dysfunction in female rats," Journal of Medicinal Food, vol. 23, no. 4, pp. 367-374, 2020.

[69] S. Ramalingam, M. Packirisamy, M. Karuppiah et al., "Effect of $\beta$-sitosterol on glucose homeostasis by sensitization of insulin resistance via enhanced protein expression of $\mathrm{PPR} \gamma$ and glucose transporter 4 in high fat diet and streptozotocininduced diabetic rats," Cytotechnology, vol. 72, no. 3, pp. 357-366, 2020.

[70] M. Kurano, K. Hasegawa, M. Kunimi et al., "Sitosterol prevents obesity-related chronic inflammation," Biochimica et Biophysica Acta (BBA)_Molecular and Cell Biology of Lipids, vol. 1863, no. 2, pp. 191-198, 2018.

[71] X. Huang, G. Liu, J. Guo, and Z. Su, "The PI3K/AKT pathway in obesity and type 2 diabetes," International Journal of Biological Sciences, vol. 14, no. 11, pp. 1483-1496, 2018.

[72] M. Ahmadian, J. M. Suh, N. Hah et al., "PPAR $\gamma$ signaling and metabolism: the good, the bad and the future," Nature Medicine, vol. 19, no. 5, pp. 557-566, 2013.

[73] R. Ramasamy, S. F. Yan, and A. M. Schmidt, "Receptor for AGE (RAGE): signaling mechanisms in the pathogenesis of diabetes and its complications," Annals of the New York Academy of Sciences, vol. 1243, no. 1, pp. 88-102, 2011. 